\title{
Structural transformations in carbon nanoparticles induced by electron irradiation
}

\author{
(C) F. Banhart
}

Z.E. Elektronenmikroskopie, Universität UIm, 89069 Ulm, Germany

The paper reviews the effects of electron irradiation in graphitic nanoparticles. Irradiation-induced atom displacements cause structural defects in the graphite lattice that forms the basis of carbon nanoparticles such as nanotubes or carbon onions. Defects of the type of non-six-membered rings induce topological alterations of graphene layers. The generation of curvature under electron irradiation leads to the formation of new structures such as spherical carbon onions or coalesced nanotubes. At high temperature, the self-compression of carbon onions can promote the nucleation of diamond cores or phase transformations of foreign materials that are encapsulated by onion-like graphitic shells. Under the non-equilibrium conditions of intense irradiation, the phase equilibrium between graphite and diamond can be reversed. It is shown that graphite can be transformed to diamond even if no external pressure is applied. All electron irradiation and imaging studies described here were carried out by in situ transmission electron microscopy.

\section{The irradiation of graphitic structures}

The irradiation of solids with high-energetic particles can lead to structural alterations if atoms are persistently removed from their lattice positions. Graphite behaves under irradiation as most metals, i. e., radiation damage is restricted to knock-on atom displacements, whereas excitations of the electron system do not lead to observable radiation effects [1]. Atoms can be displaced in a knock-on collision when the particle energy exceeds the displacement threshold energy. This energy is needed to displace an atom far enough from its position so that immediate recombination of the Frenkel pair does not occur. The minimum energy which has to be transferred to a carbon atom in graphite to induce its permanent displacement is approximately $15 \mathrm{eV}$ [2]. The corresponding energy of the irradiating particles can be calculated from the laws of momentum conservation; for example, electrons with their low mass need a threshold energy of $100 \mathrm{keV}$ to induce structural alterations of the graphite lattice. In the following we will constrain our considerations to electrons as irradiating particles because of the importance of irradiation effects in the beams of electron microscopes.

The graphite lattice consists of flat graphene layers in which each carbon atom is bound covalently to three neighbour atoms so that a network of hexagonal rings is formed. When a carbon atom is knocked from its lattice site, a monovacancy is created that is stable until recombination with a mobile interstitial atom can occur (Fig. 1, $a$ ). The diffusivity of interstitials is an exponential function of temperature, therefore the recombination dynamics in a graphitic structure under irradiation is governed by the temperature but also by the displacement rate which is proportional to the irradiation intensity. At low temperature, interstitials tend to form agglomerates between the basal layers. These agglomerates act as traps for further interstitials so that new basal layers are gradually generated [2]. This leads to a swelling of the graphite lattice in c-direction [3] and to a highly defective structure as can be seen in high-resolution electron microscopy. At temperatures above approximately
$600-700 \mathrm{~K}$, the mobility of interstitials is high enough to promote annealing with vacancies. No more clustering of interstitials can take place and the graphite lattice remains free of extended defects [4]. It is noteworthy that vacancies in graphite are immobile up to very high temperatures and play no considerable role in the defect dynamics and annealing behaviour [5]. Since graphite has a layered crystal structure, the mobility of interstitials is highly anisotropic. Opposite to earlier expectations, the migration of interstitials is not restricted to the interplanar space [2]. When an interstitial is located between the planes, the local bonding character of the surrounding atoms can change from $s p^{2}$ towards $s p^{3}$. In such a way, the interstitial forms covalent
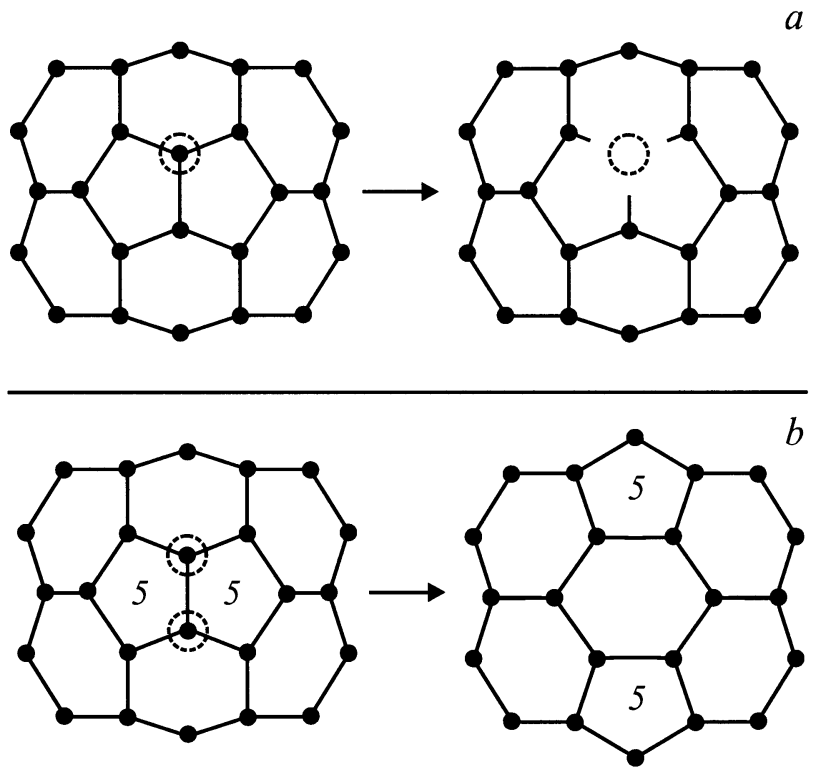

Figure 1. Vacancy formation in the graphite lattice: $a$ - the removal of one carbon atom (encircled) from a curved graphene layer creates a monovacancy that is stable, $b$ - the removal of two adjacent atoms creates a divacancy that can close via Stone-Wales rearrangement. The number of hexagons is reduced by one and the surface area is reduced. 
bonds to the adjacent planes which drastically reduces its mobility in the space parallel to the basal planes. Such an interstitial can rather migrate along the $c$-direction by exchanging position with atoms in the plane [6].

The defective graphite lattice has a peculiarity that distinguishes its behaviour under irradiation from that of most other crystals. As soon as two adjacent atoms are displaced, i. e. a divacancy is created, the surrounding atoms within the plane can rearrange bonds in such a way that the open space is immediately closed, as shown schematically in Fig. 1, $b$. These changes of bonding in a defective graphene layer are known as Stone-Wales rearrangements [7]. In such a process, hexagonal rings are converted to pentagons, heptagons, or other types of structural defects. Since the discovery of the fullerenes, it is known that non-six-membered rings introduce curvature into the otherwise flat graphene layer. Pentagons lead to positive (spherical), heptagons to negative (saddle-like) curvature, whereas appropriate combinations of both pentagons and heptagons can be used to build up a variety of models of possible topologies $[8,9]$. The irradiation-induced rearrangement of the atomic structure in graphitic networks is therefore a promising way to create new architectures in the world of carbon nanoparticles.

The first structural transformation has been achieved when graphitic structures were transformed to spherical concentric shell particles (carbon onions) [10]. The introduction of pentagons and heptagons by irradiation induces curvature in the layers which then close upon themselves by saturating all dangling bonds. However, it turned out that new structures that are created by irradiation can again be destroyed by atom displacements so that only those arrangements remain stable that are in a dynamic equilibrium under irradiation [2]. Since objects under intense irradiation are far from thermal equilibrium, the formation of ordered structures follows the principles of self-organization in dissipative systems. Apparently, the generation of carbon onions as well as their transformations under further irradiation can serve as examples of such selforganized non-equilibrium structure formation.

\section{Experimental techniques}

The investigation of irradiation-induced structural transformations of carbon nanoparticles was undertaken by in situ transmission electron microscopy. Irradiation with highenergetic electrons and imaging on an atomic scale can be carried out at the same time. However, the electron energy has to exceed the displacement threshold in graphitic structures of about $100 \mathrm{keV}$. The studies presented in the following sections were carrier out in a high-voltage electron microscope with an acceleration voltage of $1.25 \mathrm{MeV}$, installed at the Max-Planck-Institut für Metallforschung in Stuttgart. A high-temperature specimen stage allowed to vary the specimen temperature between room temperature and $1300 \mathrm{~K}$. Specialized drift compensation systems were used to obtain a point resolution of $0.12 \mathrm{~nm}$ within the full temperature range. An imaging filter system was available to carry out electron energy-loss spectroscopy in order to obtain information on bonding states and to distinguish between graphitic and diamond-like modifications of carbon.

\section{Observations and discussion}

3.1. Fullerenes. When fullerites, i.e., crystals that are built up by fullerene molecules, are exposed to an electron beam of more than $100 \mathrm{keV}$ energy, the fullerene cages are rapidly damaged by the loss of atoms. In the microscope this is seen as a collapse of the molecules which then tend to coalesce so as to form larger irregular cages [11]. In the cause of this the regular arrangement of molecules is completely lost. However, the picture can be different when individual fullerene molecules are irradiated. Under irradiation of graphitic material, the formation of single fullerene-like cages of different size is often observed. These cages are located on graphite surfaces, and they are surprisingly stable under the beam. Such a high radiation hardness was explained in terms of a mechanism where

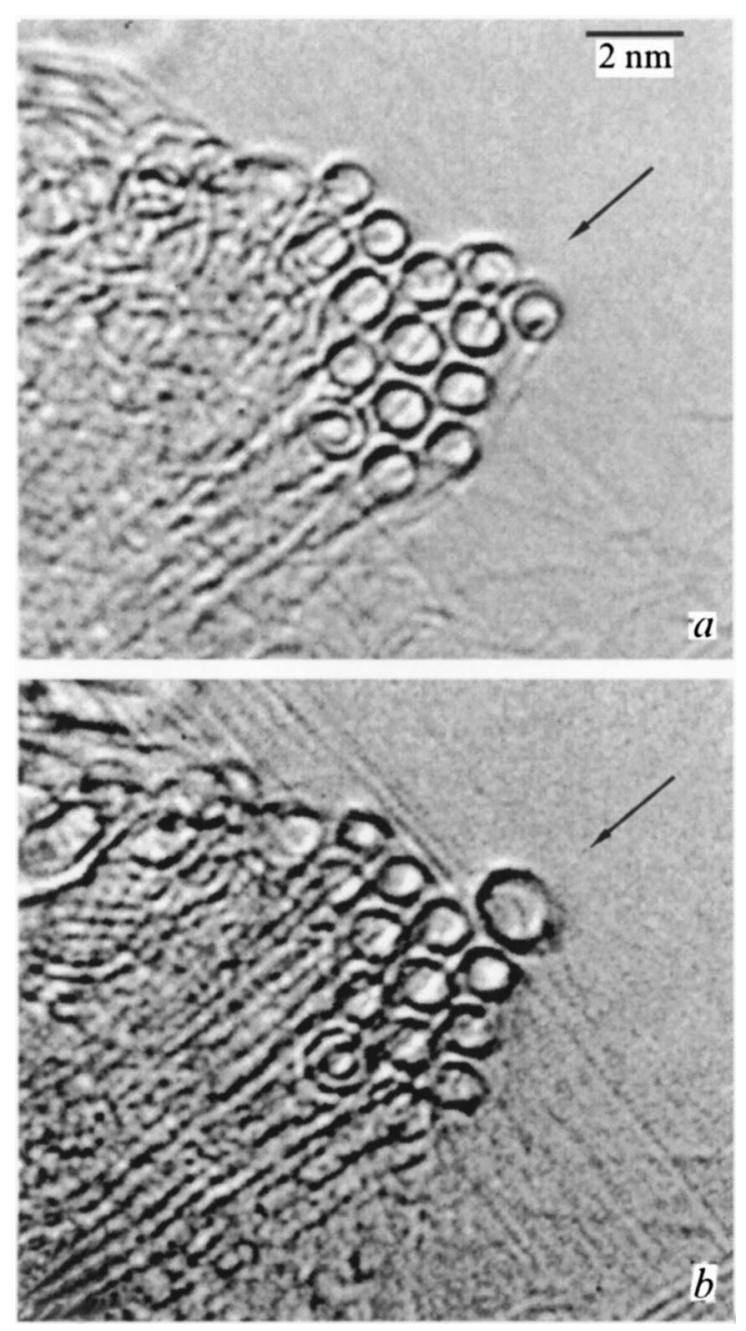

Figure 2. Coalescence of two single-walled nanotubes under electron irradiation at $1000 \mathrm{~K}$. $a$ - before, $b$ - after irradiation. The merging tubes are arrowed. 

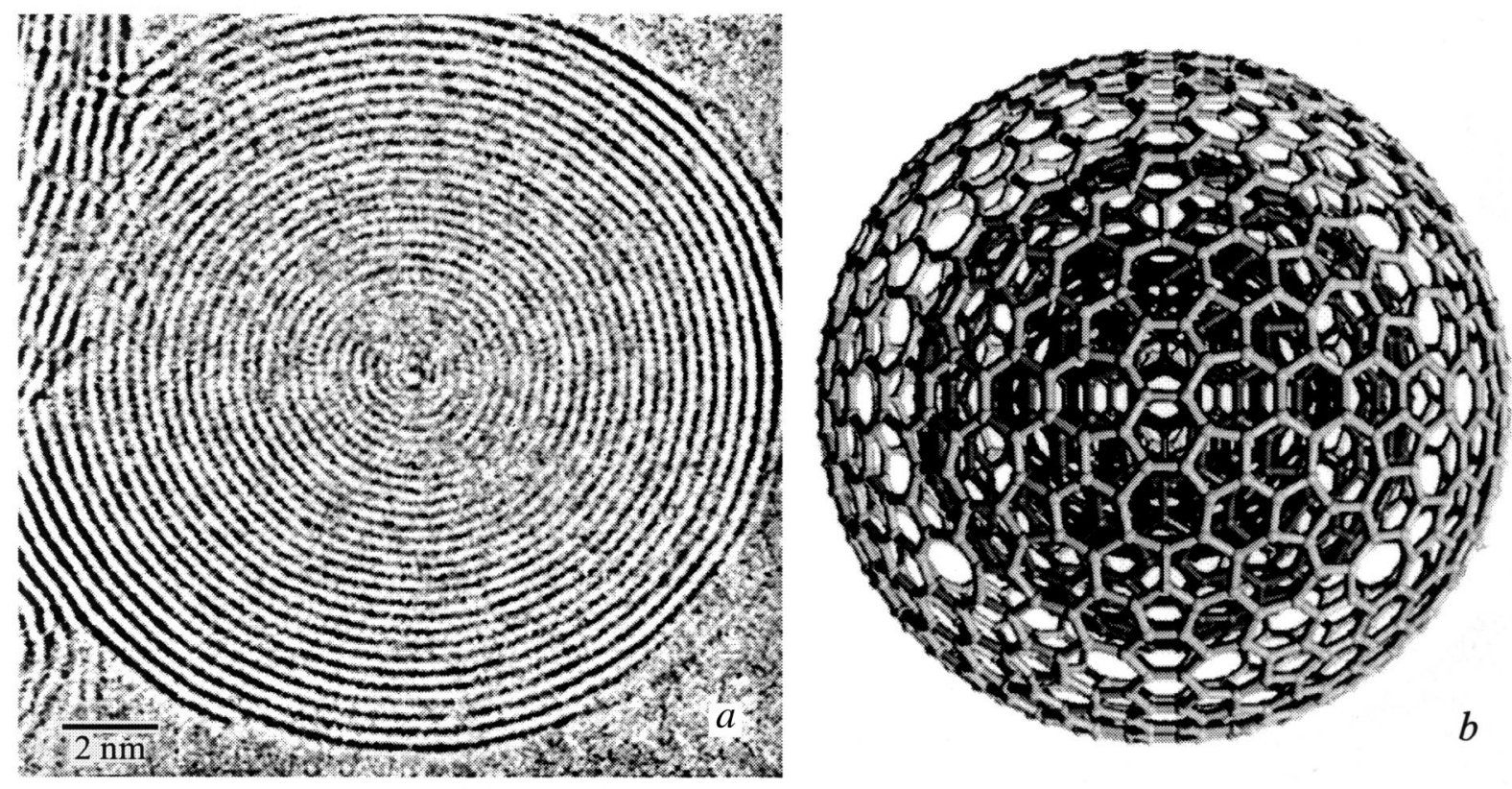

Figure 3. The structure of carbon onions. $a$ - electron microscopy image of a carbon onion, formed under electron irradiation at $1000 \mathrm{~K}$. The shells are coherent and show a decreasing spacing towards the centre. $b$ - model structure of a carbon onion with three shells (not at the same scale as $(a)$ ). Pentagons and heptagons ensure uniform curvature (courtesy by M. Terrones).

vacancies in the fullerene shell can recombine with free carbon atoms that are available on the graphite surface [11].

3.2. Carbon nanotubes. Multi-walled carbon nanotubes show the genaral behavior of graphite when irradiated with an electron beam. At low temperature, radiation defects accumulate and the graphitic planes curve and bend; this can eventually lead to the complete transformation of the tube to a carbon onion [10]. At temperatures above $600 \mathrm{~K}$, the graphitic lattice of the tubes remains free of extended defects and the tubes bend but do not transform to onionlike structures.

Single-walled carbon nanotubes are very sensitive to electron irradiation at low temperature. Shrinking and collapse of the tubes occurs, leading to a necklace-like shape and eventually complete disintegration [12]. As other graphitic structures, single-walled nanotubes show a much improved stability when irradiated at higher temperature. The irradiation of a bundle of single-walled nanotubes can lead to the coalescence of two adjacent tubes so as to form a tube of double diameter [13]. As shown in Fig. 2, such a coalescence can be monitored in situ in the electron microscope when curved bundles of tubes are locally aligned with their axes parallel to the viewing direction (resp. the optical axis of the microscope) so that they are imaged in cross-section. Molecular dynamics calculations show that the coalescence of adjacent tubes can only occur if the tubes have the same chirality [13].

3.3. Carbon onions. The most spectacular irradiation effect of carbon nanoparticles is the transformation of graphitic material to spherical concentric-shell carbon onions [10]. Almost all graphitic precursors such as soot particles, nanotubes, or any disordered graphitic filaments show this transformation when irradiated with an electron beam of sufficient intensity. The structure of carbon onions has been modelled by combining pentagons, hexagons, and heptagons in each shell (Fig. 3) [8,14]. In such combinations, a uniform spherical curvature of the shells can be achieved without altering the bond length between two carbon atoms $(0.14 \mathrm{~nm})$. Complete spherical closure with saturation of all dangling bonds leaves a particularly stable structure because further atom displacements just rearrange defects but do not change the curvature anymore.

The formation of carbon onions under irradiation at higher specimen temperature leads to perfectly coherent graphitic shells without extended defects. Such carbon onions show a spacing between the shells which is smaller than the equilibrium distance between basal planes in graphite $(0.335 \mathrm{~nm})$. Furthermore, the spacing decreases from the surface of the onion towards its centre. This self-compression of carbon onions under irradiation has been explained in terms of surface tension of the outer shells due to the sputteringinduced loss of atoms and successive closure of the shells around divacancies. The migration dynamics of interstitials through the onion, following the pressure gradient towards the surface, can explain why the pressure is not isostatic but increases towards the centre [15]. The high mechanical strength of graphene layers makes the shells extremely rigid and allows the generation of enormous pressure inside the onion. The pressure can reach values that are high enough to promote the nucleation of a diamond crystal in the core of the onion [16]. The phase transformation to diamond is facilitated by the high curvature of the innermost shells, shifting the bonding character from $s p^{2}$ towards $s p^{3}$. 


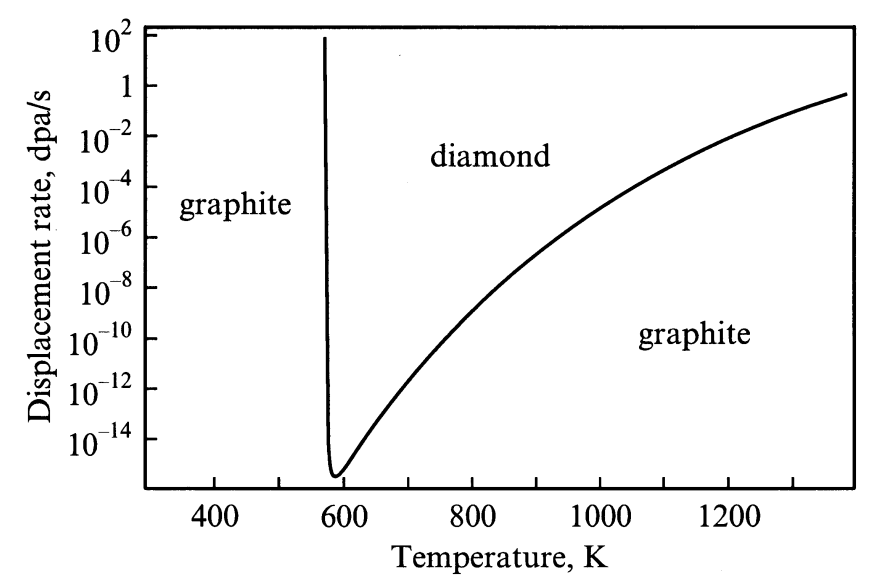

Figure 4. Non-equilibrium phase diagram of carbon under highenergetic electron irradiation. The displacement rate (proportional to the beam intensity) is plotted as a function of temperature. A ratio of displacement cross-sections in graphite and diamond of $\sigma_{g} / \sigma_{d}=2.6$ has been assumed.

3.4. Transformation of graphite to diamond under irradiation. The phase equilibrium between graphite and diamond is determined by the difference in free enthalpy between the two phases. Close to thermal equilibrium, graphite is thermodynamically favoured at low pressure and thus represents the stable phase of carbon. Diamond is the stable high-pressure phase but can exist in a metastable state at low pressure because the transformation to graphite is kinetically suppressed. The transformation of graphite to diamond requires the application of high pressure and high temperature and, to achieve a reasonable yield of diamond, the presence of metallic catalysts [17].

The function of carbon onions as pressure cells for diamond nucleation has shown a new way of transforming graphite to diamond. It has been observed that diamond cores in carbon onions, once nucleated, grow under sustained electron irradiation. Growth of the diamond crystals at the expense of the surrounding graphite shells continues until the onions have wholly transformed to diamond, although no more pressure is applied to the system at the later stage of the process. This observation has motivated further studies in which a flat interface between graphite and diamond was irradiated with an electron beam at different beam intensities and temperatures. These experiments were carried out in the heating stage of an electron microscope; no external pressure was applied. It turned out that within a limited temperature range the phase equilibrium can be reversed so that diamond becomes the stable phase [18]. A theoretical treatment of the phase equilibrium between graphite and diamond under irradiation could explain the observations quantitatively [19]. The theory is based on the well-known fact that the radiation hardness of diamond exceeds that of graphite, i.e., that the atom displacement rate in graphite is higher than in diamond (at the same conditions of irradiation). When a carbon atom at the interface between the two phases is displaced, its survival time is longer if it aggregates to the diamond crystal. Thus, a net growth of diamond at the expense of graphite occurs which depends on the displacement rate that is again proportional to the beam intensity. However, at high temperatures thermal jumps of atoms across the interface prevail and restore thermal equilibrium so that graphite becomes the stable phase again and grows at the expense of diamond. In such a way, a non-equilibrium phase diagram of carbon can be obtained such as shown in Fig. 4. The only adjustable parameters in the model are the displacement threshold energies of graphite and diamond which are not known with high accuracy from earlier studies. The adjustment of these parameters within a reasonable range has led to an excellent match of experimental and theoretical results [20]. These experiments have thus demonstrated for the first time that the transformation of graphite to diamond is feasible at zero pressure under the non-equilibrium conditions of intense particle irradiation.

3.5. Metal-carbon nanoparticles. The encapsulation of crystalline metals in graphitic shells, either in nanotubes or in onions, is a subject of considerable interest [21]. Among techniques such as coevaporation of carbon together with metals at high temperature, irradiation of metal-carbon composites has shown to produce metal crystals that are completely encapsulated by graphitic shells [22]. This process occurs similar to the formation of carbon onions under irradation but with the difference that here graphitic shells wrap around nanometre-sized metal crystals so as to form onion-like spherical graphitic particles with metal cores. Examples of the encapsulated transition metals Fe, Co, and $\mathrm{Ni}$ are shown in Fig. 5.

A peculiarity of these encapsulates is that the graphitic shells self-compress under electron irradiation so that considerable pressure is exerted on the metal crystal in the core. As a consequence, atoms at the metal-graphite interface can leave the metal crystal and migrate along the pressure gradient towards the surface of the graphitic shell. This leads to a gradual shrinkage of the metal crystal inside the compressing shells until the crystal has completely vanished [22]. This phenomenon is of interest because it allows the study of the migration of foreign atoms through graphitic structures. Apparently, foreign atoms occupy irradiation-induced vacancies and migrate in $c$-direction via an exchange of position with carbon atoms. Most foreign atom species are unstable on lattice sites of graphite and tend to migrate rapidly, however some elements form configurations with graphite that are at least temporarily stable. It has been observed that nickel atoms form metastable ordered arrangements during their migration through the graphitic shells [23]. This can be seen on the right hand side in Fig. 5 where the shells are modified due to the presence of $\mathrm{Ni}$ atoms.

The action of graphitic shells as pressure cells for encapsulated foreign materials is highly useful for the study of pressure-induced phase transformations in small crystals. Very recent experiments show that the melting behaviour of low-melting metals is completely altered when they are encapsulated in graphitic shells [24]. 

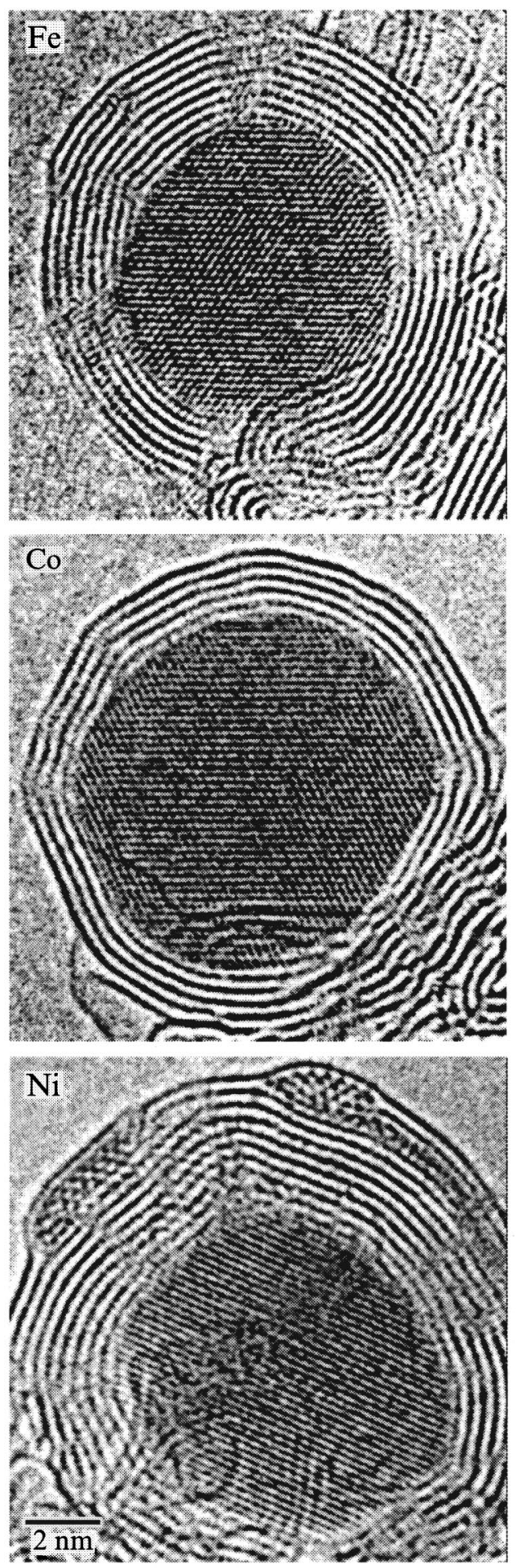

Figure 5. Metal crystals that were encapsulated by graphitic shells under electron irradiation. Examples for $\mathrm{Fe}, \mathrm{Co}$, and $\mathrm{Ni}$ crystals are shown.

A variety of new and unexpected phenomena resulted from the irradiation of graphitic nanoparticles with highenergetic electrons. Although the atoms are displaced randomly by knock-on collisions with electrons, highly ordered structures can emerge from such non-equilibrium processes. The introduction of defects into graphene layers leads to curvature and to a change in topology of the structures. Perfectly spherical carbon onions have shown to be the most stable arrangements of graphitic carbon under irradiation. The self-compresstion of carbon onions can be made use of to nucleate diamond or to exert pressure on foreign materials that are encapsulated under irradiation in the cores. The study of carbon structures under irradiation has led to the discovery that the non-equilibrium conditions of irradiation can reverse the phase stability between graphite and diamond and to enable us to transform graphite to diamond without applying pressure.

The author is grateful to Y. Lyutovich, M. Zaiser, M. Terrones, P.M. Ajayan, J.-C. Charlier, T. Füller, M. Zwanger, $\mathrm{Ph}$. Kohler-Redlich, and A. Seeger who have contributed to the work presented in this review during years of excellent collaboration.

\section{References}

[1] B.T. Kelly. Physics of Graphite. Applied Science. London (1981).

[2] F. Banhart. Rep. Prog. Phys. 62, 1181 (1999).

[3] J. Koike, D.F. Pedraza. J. Mater. Res. 9, 1899 (1994).

[4] F. Banhart, T. Füller, Ph. Redlich, P.M. Ajayan. Chem. Phys. Lett. 269, 349 (1997).

[5] P.A. Thrower, R.M. Mayer. Phys. Stat. Sol. (a) 47, 11 (1978).

[6] M. Heggie, B.R. Eggen, C.P. Ewels, P. Leary, S. Ali, G. Jungnickel, B. Jones, P.R. Briddon. In: Recent Advances in the Chemistry and Physics of Fullerenes and Related Materials / Ed. by K.M. Kadish, R.S. Ruoff. The Electrochemical Society, San Diego (1998). Vol. 6. P. 60.

[7] A.J. Stone, D.J. Wales. Chem. Phys. Lett. 128, 501 (1986).

[8] H. Terrones, M. Terrones, W.K. Hsu. Chem. Soc. Rev. 24, 341 (1995).

[9] M. Terrones, H. Terrones. Fullerene Sci. Technol. 4, 517 (1996).

[10] D. Ugarte. Nature 359, 707 (1992).

[11] T. Füller, F. Banhart. Chem. Phys. Lett. 254, 372 (1996).

[12] P.M. Ajayan, V. Ravikumar, J.-C. Charlier. Phys. Rev. Lett. 81, 1437 (1998).

[13] M. Terrones, H. Terrones, F. Banhart, J.-C. Charlier, P.M. Ajayan. Science 288, 1226 (2000).

[14] K.R. Bates, G.E. Scuseria. Theor. Chem. Acc. 99, 29 (1998).

[15] M. Zaiser. Mater. Res. Soc. Symp. Proc. 540, 243 (1999).

[16] F. Banhart, P.M. Ajayan. Nature 382, 433 (1996).

[17] G. Davies. Properties and Growth of Diamond. INSPEC, London (1994).

[18] Y. Lyutovich, F. Banhart. Appl. Phys. Lett. 74, 659 (1999).

[19] M. Zaiser, F. Banhart. Phys. Rev. Lett. 79, 3680 (1997).

[20] M. Zaiser, Y. Lyutovich, F. Banhart. Phys. Rev. B62, 3058 (2000).

[21] M. Terrones, N. Grobert, W.K. Hsu, Y.Q. Zhu, W.B. Hu, H. Terrones, J.P. Hare, H.W. Kroto, D.R.M. Walton. Mater. Res. Soc. Bull. 24, 43 (1999).

[22] F. Banhart, Ph. Redlich, P.M. Ajayan. Chem. Phys. Lett. 292, 554 (1998).

[23] F. Banhart, J.-C. Charlier, P.M. Ajayan. Phys. Rev. Lett. 84, 686 (2000).

[24] M. Terrones, F. Banhart, to be published. 